\title{
MarinEye - A tool for marine monitoring
}

Alfredo Martins ${ }^{\#+}$, André Dias ${ }^{\#+}$, Eduardo Silva ${ }^{\#+}$, Hugo Ferreira ${ }^{\#}$, Ireneu Dias ${ }^{\#}$, José Miguel Almeida ${ }^{\#+}$, Luís Torgo ${ }^{\# \sim}$, Marco Gonçalves ${ }^{\#}$, Maurício Guedes ${ }^{\#}$, Nuno Dias ${ }^{\#+}$, Pedro Jorge ${ }^{\#}$, Ana Paula Mucha*, Catarina Magalhães*, Maria de

Fátima Carvalho*,Hugo Ribeiro*, C. Marisa R. Almeida*, Isabel Azevedo*, Sandra Ramos*£ ,Teresa Borges* , Sérgio Miguel Leandro ${ }^{\circ}$, Paulo Maranhão ${ }^{\circ}$, Teresa Mouga ${ }^{\circ}$, Roberto Gamboa $^{\circ}$, Marco Lemos $^{\circ}$, Antonina dos Santos $^{\$}$, Alexandra Silva ${ }^{\$}$, Bárbara Frazão e Teixeira ${ }^{\$}$, Cátia Bartilotti ${ }^{\$}$, Raquel Marques\$ ${ }^{\$}$ Sónia Cotrim ${ }^{\$}$

${ }^{\#}$ INESC TEC - INESC Technology and Science, Porto Portugal ${ }^{+}$ISEP - School of Engineering, Polytechnic Institute of Porto, Porto, Portugal

*CIIMAR - Interdisciplinary Center of Marine and Environmental Research, University of Porto, Portugal

${ }^{£}$ Institute of Estuarine and Coastal Studies, University of Hull, Hull HU6 7RX, UK

$\sim$ FCUP -Faculty of Sciences of University of Porto, Porto, Portugal

${ }^{\circ}$ MARE - Marine and Environmental Sciences Centre, Escola Superior de Turismo e Tecnologia do Mar - Instituto Politécnico de Leiria, Leiria, Portugal

${ }^{\$}$ IPMA - Portuguese Sea and Atmosphere Institute, Lisbon, Portugal

\begin{abstract}
-
This work presents an autonomous system for marine integrated physical-chemical and biological monitoring - the MarinEye system.

It comprises a set of sensors providing diverse and relevant information for oceanic environment characterization and marine biology studies. It is constituted by a physicalchemical water properties sensor suite, a water filtration and sampling system for DNA collection, a plankton imaging system and biomass assessment acoustic system.

The MarinEye system has onboard computational and logging capabilities allowing it either for autonomous operation or for integration in other marine observing systems (such as Observatories or robotic vehicles. It was designed in order to collect integrated multi-trophic monitoring data.
\end{abstract}

The validation in operational environment on 3 marine observatories: RAIA, BerlengasWatch and Cascais on the coast of Portugal is also discussed.

\section{INTRODUCTION}

The world's oceans are central to the habitability of our planet, although the dynamics of its environments, with an integrated view of all their components, is largely unknown with vast unexplored regions and ecosystems that have yet to be characterized. Growth in our understanding of the complex exchange among processes throughout ocean basins is severely limited by the paucity of infrastructures able to support sustained and interactive observations of the biological, chemical, physical, atmospheric and geological processes. Because all these processes interact in the ocean in complex ways, developing a more fundamental scientific understanding of these relationships requires new and transformational approaches to ocean observation.

Marine organisms exert tremendous power over the planet, and changes in the smaller-size fraction of the ocean biological compartment can have a boomerang effect, bringing with them changes in the primary productivity of the oceans, changes in fishery catch potential, patterns of harmful algal bloom occurrences, dispersal of invasive species, and many other shifts in marine habitats around the world [1]. Understanding how these complex marine ecosystems work requires a holistic approach, with application of a range of technologies including genomics, high-throughput image, hidroacoustics and physical and chemical sensors[2]

While ocean observation technologies and programs have made considerable progress in advancing physical and chemical observing systems, bio-geochemical and biological ocean monitoring is still under-represented and not implemented on ocean observatories [3] [4].

In this work we developed a concept of an autonomous system for marine integrated physical-chemical and biological 
monitoring - MarinEye. This system will address the urgent needs of ocean observatories by monitoring integrated biological variables in contextualized environmental data (physical-chemical variables), providing key data to respond to time sensitive ocean issues, essential to evaluate the environmental status of marine ecosystems. Additionally, more readily available oceanic data are needed to assist the public and private sectors in risk management connected with the sea such as the weather, major transport accidents, marine pollution, as well as to support decisions related to marine activities in the energy and aquaculture sectors.

MarinEye will combine imaging, acoustic, sonar, fraction filtration systems coupled to DNA/RNA preservation) and sensors (targeting physical-chemical variables) technologies in a modular, compact system that can be deployed on fixed and mobile platforms. This device will strengthen the knowledge of oceanic processes and the observational infrastructures, constituting an important complement to the already existent ocean observatories by providing novel integrate data not currently supplied, being an extremely important contribution for the consolidation of infrastructures dedicated to the observation of the marine environment.

\section{SYSTEM REQUIREMENTS}

MarinEye pretends to be an innovative multitrophic system with adequate sensors, sufficient autonomy and robustness, including physical, chemical and biological monitoring, to assess the state of the marine environmental ecosystems for coastal waters and remote oceanic areas such as the deep sea. MarinEye is expected to provide information that could not be obtained by satellites or aircrafts, by increasing the monitoring capacities carried out by vessels, AUVs, oceanic and coastal platforms (fixed or mobile). The development of the proposed multitrophic autonomous monitoring system capable of acquiring biological data at the same spatio-temporal resolution than the physical and chemical data will represent a great advance in integrated ocean data generation with an important impact for any monitoring program and for the implementation of the Marine Strategy Framework Directive (MSFD).

The operational conditions of the different modular components of MarinEye will be defined based on the characteristics of three marine observatories. These will include remote oceanic areas, (platform buoy of the RAIA observatory, located in the slope of the continental shelf of the North West Iberian Atlantic margin, more than 50 miles offshore http://www.marnaraia.org/), Marine Protected Areas
(BerlengasWatch observation program) and coastal upwelling areas (CascaisWatch observation program; http://www.st.nmfs.noaa.gov/plankton/time-

series/site_iberian-portugal-cascais/). The CascaisWatch is a long term monitoring site maintained by IPMA. It is located at $38^{\circ} 40^{\prime} \mathrm{N} 09^{\circ} 26.2^{\prime} \mathrm{W}$ approximately $4 \mathrm{~km}$ offshore Cascais (40 $\mathrm{km}$ west of Lisbon), in a water depth of $38 \mathrm{~m}$. The hydrography of Cascais bay is influenced by coastal morphology, the bottom topography (submarine canyons of Lisbon and Setúbal), and the discharge of freshwater from the Tagus River. This site is under the influence of the Eastern North Atlantic Upwelling System in spring and summer. The time series started in 2005, on a monthly basis, and includes temperature, salinity, and Chlorophyll a measurements, zooplankton sampling (oblique and horizontal hauls), phytoplankton (Niskin bottle) and chemical parameters. The Berlengas site is a station of the time-series BerlengasWatch oceanographic observation program of IPMA, with the collaboration of MARE-IPLeiria - Polytechnic Institute of Leiria. The station is located off the Peniche coastline, at $39^{\circ} 25^{\prime} 940 \mathrm{~N}, 9^{\circ} 30^{\prime} 608 \mathrm{~W}$. Berlengas site is located in a zone with a temperate maritime climate and is influenced by seasonal coastal upwelling controlled by the atmospheric circulation associated with the Azores anticyclone. It is located at the top of the escarpment of the Nazare Canyon, one of the most important submarine canyons in the international context and in a transition zone between the Mediterranean and European sub regions.

Both locations contributes to the remarkable productivity and diversity of marine species and habitats and to a landscape unique in the Portuguese coast.

RAIA observatory is an extended grid of oceanographicmeteorological automated observations on the continental shelf in the trans-frontier region of Northern Portugal and Galicia (www.marnaraia.org). This ocean observatory integrates platform and coastal oceanographic-meteorological buoys located at the North West Iberian Atlantic margin, an area of high biodiversity and of great economic importance (increasing marine transit, fishing resources). RAIA is based on the monitoring and forecast of the ocean environment through the use of numerical models and the real-time data derived from oceanographic-meteorological platforms. Realtime meteorological, oceanographic and chemical data are measured at $10 \mathrm{~min}$ frequency, namely air temperatures, humidity wind, sea temperature, salinity, density and currents at several depths and oxygen concentrations.

Once operational, data obtained from these three marine observatories will be disseminated through the National 
Information Sharing Environment (NISE) contributing to the Integrated Maritime Surveillance and Monitoring (NIPIM@R), contributing to the maintenance of the Long Term Ecological Research Network (https://www.lternet.edu/).

Current ocean observation data rely in a wide variety of systems ranging from local deployed sensors to relatively large cabled ocean observatories. Data can also be collected from fixed data acquisition nodes or deployable systems either in standard vessel based campaigns or with autonomous robotic systems [5].

These wide ranges of operational requirements were also taken into account in the development of the MarinEye system. In order to be able to accommodate various operating modes, autonomy issues were addressed, namely on the energy aspects and on the autonomous data logging capabilities.

The possible application in mobile robotic platforms[6], [7] and also on other integrated structures [8],[9] also imposed requirements in terms of dimensions and weight along with interface constraints.

Both autonomous logging and external control modes were considered in the system design. A modular approach was taken in order to provide flexibility for possibly tight restrictions such as in the integration on small robotic vehicles such as AUVs. When integrated in underwater robotic vehicles particular sensor transducers can be physically separated from on-board logging and usually there is no need of autonomous power (since it is available from the vehicle itself). Depending on the level of integration, this however can change for the cases when the MarinEye is used as an independent payload. In addition, the modular approach allows for possible multiple components configurations and future expansion.

On board processing capabilities allow both for multiple mission specific data logging strategies, enabling the reducing of logged data and extended operational time. It also endows the vehicle with the computational power not only to preprocess sensor data but also to allow for complex mission programming.

\section{MARINEYE SYSTEM}

\section{A. System architecture}

MarinEye comprises a set of modular sensory subsystems assembled in a single multithrophic system for integrated biological marine studies (see Figure 1).
The main sensing components of the system are a water parameter sensor suite, a water filtration fraction system for RNA/DNA sampling, plankton imaging system and an acoustic system for environment acoustics logging, biomass, and abundance and diversity measurements.

These sensing and data measurement sources are integrated in the system providing a spatial and time synchronous data logging capability. This diverse set of information gathering capabilities also allows for complex mission oriented sampling strategies. All the MarinEye subsystems are controlled by a low power embedded main CPU. This allows not only for time synchronization and global system control, but also for local processing allowing detection of relevant events triggering specific measurement procedures, such as for ex: the collection of a DNA water sample upon a detection of the imaging system.

The main CPU is a high performance multicore low power ARM based system (Odroid XU4). It also controls the global power management in order to switch on and off subsystems to reduce power consumption for extended periods of time. It also provides the central data logging capabilities, mission profile definite, global system control and external interface (either to user based PCs or to other systems when integrated in robotic vehicles or other infrastructures).

A set of onboard batteries provides power to all the systems when MarinEye is used in standalone mode. Another option is the supply of the main power being provided externally when convenient (such as when integrated in autonomous underwater vehicles).

A modular approach has been taken in the development of the system (see Figure 1) with all the sensors and different subsystems having a local microcontroller providing dedicated control with a clear digital interface to the central CPU. This modularity reduces computational load and complexity on the main CPU and provides a higher level abstraction of the separate sensors. In addition the modularity advantages also provide ease setup of future new functionalities and sensors.

A GPS and wireless communications link is also integrated. Although not useful for underwater, the wireless communication link allows for ease of data transfer and communication with the main CPU when at surface and thus brings field operational advantages. The GPS is used both for internal time synchronization when at surface and also to provide an optional location retrieval information when the system is lost (with the position information sent through and additional optional satellite or cellular wireless link). 


\section{A. Physical-chemical modular multi-sensor system}

The multi-sensor suite subsystem provides information for the physical-chemical characterization of the water environment. From a biological point of view, the water properties have a relevant impact on the ecosystem and on the particular studies to be performed, and are of crucial nature.

These properties are typically assessed in oceanographic campaigns or measurements and can either be obtained from direct sensor readings (with multiparametric or single parameter probes) or from posterior lab analysis of water samples.

Classic sensor setups will include CTDs, oxygen, $\mathrm{pH}$, fluorometer, turbidity sensors or other chemical parameter sensors.

Taking into account that this system aims to be used in a wider range of marine biology studies, the inclusion of the most relevant sensors has to be considered. Additionally the system must be able to be extended or configured with a particular suite of sensors.

The approach taken in the development of the water physical-chemical sensor subsystem was to incorporate from the start a basic configuration with the sensors more relevant for the requirements defined in the test sites. It was also defined that additional standard sensor interfaces would be available for expansion allowing the plug in of particular sensors.

As referred in the system architecture description, the main computational system has a set of standard communication interfaces (namely serial and Ethernet ones) that are physically available. Power delivery for additional sensors is also included in the global power management system. Integration of extra sensors will require only cable adaptation and can easily be performed.

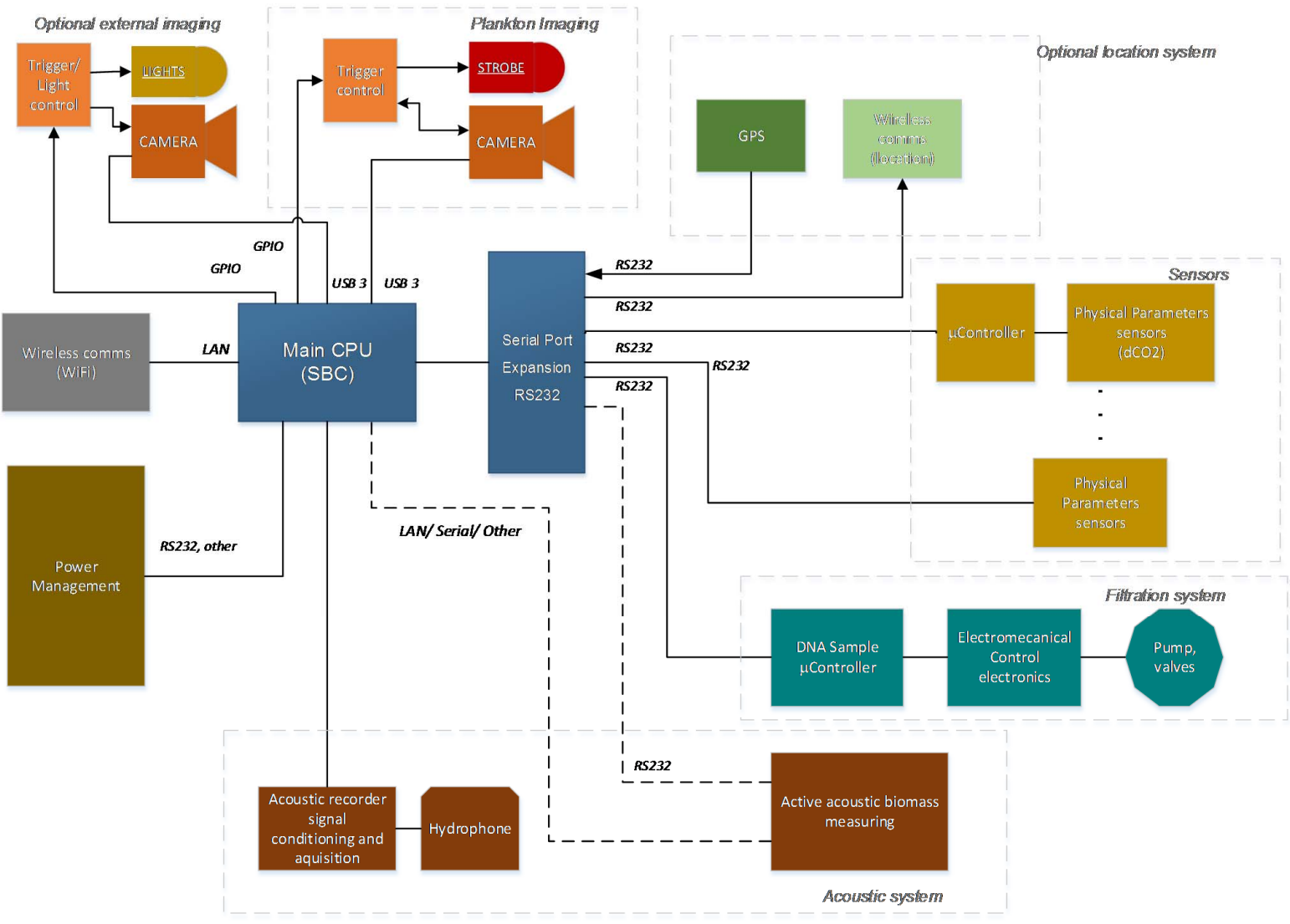

Fig 1. MarinEye system architecture 
The basic set of sensors included comprises a CTD, and sensors for $\mathrm{pH}$, dissolved oxygen, salinity and temperature, as well as a sensor for dissolved $\mathrm{CO} 2$ (dCO2) With the exception of the dCO2 sensor, all probes are standard commercial-of-theshelf (COTS) systems. The dCO2 one is a custom developed sensor.

This novel dCO2 sensor based on fiber optical sensing technology was integrated not only because of the relevance of $\mathrm{dCO} 2$ in the water characterization essential to evaluate the response to anthropogenic inputs of $\mathrm{CO} 2$ and to understand the changing chemistry and the impacts of ocean acidification on marine ecosystems, but also to validate the technologic approach. In fact, $\mathrm{dCO}_{2}$ is a key variable for ocean ecology and biogeochemistry. This variable allows the quantification of a diverse and crucial set of ecosystems processes, such as the magnitude and variability of net community and export production, the detection of the impact of global warming on ocean biogeochemistry and circulation, the assessment of changes in low oxygen regions as well as estimations of the oceanic uptake of anthropogenic $\mathrm{CO}_{2}$ [10]. In addition, systematic and accurate measurements of $\mathrm{dCO}_{2}$ are also essential to document the evolving response of the ocean to anthropogenic inputs of $\mathrm{CO}_{2}$ and to understand the changing chemistry of the oceans and the impacts of ocean acidification on marine ecosystems.

This optical sensing technology, also follows a modular approach, combining a low cost optoelectronic platforms with capability to interrogate different sensitive layers, offering the possibility to incorporate new developments enabling additional chemical parameters to be added to the sensors system

The dCO2 sensing system developed, is based on a proprietary sensitive layer that experiences both colorimetric and refractometric changes in the presence of carbon dioxide. Based on the polymerization of the sensitive molecules, it avoids the use of a support matrix, and can operate with very thin films, resulting in fast response times and ability to be coated over complex geometries. This allows great flexibility in the design of the sensing probe that can be implemented using different types of optical fibers, or planar platforms. The sensor probe is interrogated using multiple LED arrangements, and PWM which enables a self-referenced interrogation scheme using a single photodetector. Adjustment of the optoelectronic components, will accommodate newly developed sensitive membranes, enabling multiparameter chemical sensing..

\section{B. Water filtration system}

The insitu DNA filtration system developed for MarineEye allows the collection of multiple filtrated planktonic samples of different size fractions (higher than $0.8 \mu \mathrm{m}$, covering all unicellular Eukaryotes, and higher than $0.2 \mu \mathrm{m}$, covering prokaryotes) and store them in an adequate preservative solution that allows long-term storage for further genomic (DNA and RNA) analysis. The biological sampling will be synchronized in space and time with all the other information collected by the system (namely imaging and water parameter data).

This filtration system comprises a set of filters that are used to concentrate planktonic samples under aseptic conditions which are subjected afterwards to the immersion in a DNA/RNA preserving fluid for posterior community genomic and/or metagenomic analysis.

Although water samplers are common instruments in marine studies, there are relatively few in-situ autonomous water microbial community samplers for posterior genomic community analysis [11], [12] [13].

The proposed system uses a set of standard filters Sterivex $^{\mathrm{TM}}-\mathrm{GV} 0.22 / 0.45 \mu \mathrm{m}$, PVDF, gamma irradiated (Merck, Millipore) to concentrate planktonic samples

Compared with traditional water samplers, in-situ filtration systems such as the one developed in this work allow processing of large volumes of water with the final sample having a reduced volume thus allowing for flexible operation modes and integration in different systems (such as AUVs, fixed nodes, etc).

The Sterivex filter was chosen since it is a standard solution for seawater filtration in biological studies [14] The possibility of replicating the established laboratory microbial filtration protocol in-situ, clearly provides a sound design approach allowing for comparative system analysis and verification.

From a mechanical design perspective a fixed set of filters (contained in a replaceable cartridge with 15 filters, in yellow on Figure 2) is fed through a distribution manifold (with selection valves) by a water pump. In addition a DNA/RNA preservation fluid reservoir (in green) is also connected to the system. 


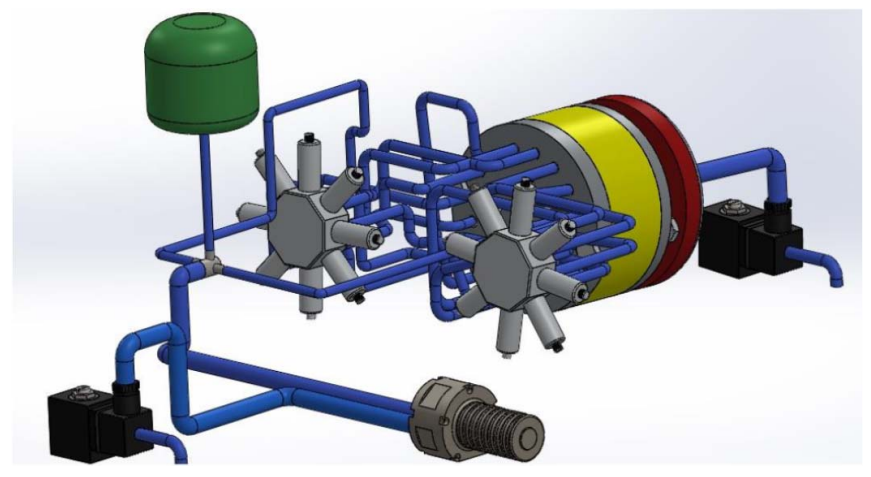

Fig. 2 - Water filtration assembly

Sample Sterivex selection is made through a set of selection valves (two manifolds per cartridge, providing 8 outputs each with one of them being used for flushing purposes.)

When sampling is to be performed, external sea water is pumped through the system in order to clean internal components (piping, pump, and valves). After this, the water is directed to pass in the selected filters. The filter clogging is detected and determines the end of the filtration process. In sequence the water in the filter capsule is removed and the capsule is filled with preserving fluid. Finally the capsule is sealed.

The system is capable of simultaneously filter multiple samples in parallel allowing for sampling replication, reducing sampling errors. In addition, in situ filtration and sample preservation will guaranty a total absent of sample transformations and cross contaminations due to sampling handling and storage, which greatly limited the precision of actual observations.

The DNA sequencing process is performed externally in the laboratory For lab processing the samples cartridge is retrieved from the system and individual filters processed externally.

Depending on the physical space available it is possible to add multiple cartridges allowing for system expansion and reconfiguration for the number of filters available for the mission.

The dedicated microcontroller unit is responsible for the pump and valve control. It processes the flow and pressure sensor data to determine the end of the filtration process and register relevant information related with the sample (such as the volume of water filtered per sample, time to filter clogging, index of filters etc). The sampling process can be controlled either based on time and volume of water to filter or on detection of the filter clogging. This embedded processor will provide the control and data interface of the filtration and sampling subsystem to the main MarinEye computational unit that is responsible for the synchronous registering of data and thus of associating the samples taken with the other information (such as water physical-chemical parameters, plankton imaging or other information).

\section{Imaging system}

One of the main MarinEye subsystems is the imaging system. This system has two main objectives: (i) to collect highresolution images of the planktonic organisms from a high range of plankton group sizes (starting detection at $20 \mu \mathrm{m}$ ) and (ii) to provide information suitable for estimating the overall plankton (of particular groups) abundance in the area. This highresolution imaging system allows rapid, quantitative and noninvasive plankton sampling and ultimately provides valuable data for structural and functional assessments of marine plankton.

In addition to plankton imaging (identification of main groups and estimation of abundance) it is often useful to add standard digital images of the environment. These can be used either to further increase the general underwater environment awareness and/or to take snapshots of large marine specimens, such as fish or marine mammals.

The primary objectives for the imaging system will be achieved with dedicated cameras and optical setup coupled with synchronous illumination. This general approach is also used in some of the currently documented systems in the literature (e.g. OPC, LOPC, VPR, SIPPER, ZOOVIS and UVP, revised in[15] and ISIIS[16]). The "standard" underwater image capture is performed with an additional camera and lighting setup specifically for this purpose.

From a physical point of view due to the multiple usages of the MarinEye system In particular, the tradeoffs between imaged volume and distance were performed taking into account the possible integration on AUVs in addition to standalone or towed solutions. 


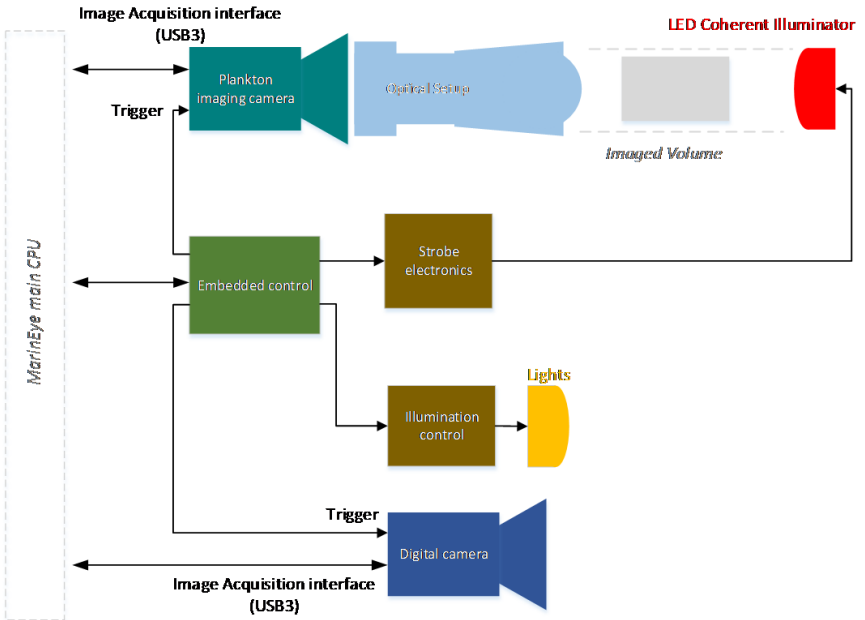

Fig. 3 - Imaging subsystem architecture

For plankton imaging, a telecentric lens is adopted in conjunction with a coherent illumination strobe. In conjunction with a $5 \mathrm{Mpixel}$ camera this system provides a $13 \mu \mathrm{m}$ of pixel resolution.

The additional imaging setup, is constituted also by a 5Mpixel camera coupled with a motorized zoom lens. An external global illumination strobe provides scene illumination. The variable zoom allows for multiple imaging options, ranging from small water volume and larger plankton sizes imaging to wide environmental images.

\section{Acoustic system}

In order to target the higher levels of the trophic chain, namely fishes and mammals, MarinEye provides an acoustic based system coupled with the water analysis and biological imaging subsystems. This subsystem has two main objectives: to record acoustic noise (with particular emphasis on biological generated signals, such as marine mammals) and to assess the presence of fish schools, number and size of fishes in the water column, and estimation of their biomass.

These two objectives are performed as can be observed in Figure 1 through two separate subsystems. The marine sound recording is performed by a hydrophone covering the frequencies of interest $(10 \mathrm{~Hz}-200 \mathrm{KHz})$ with the appropriate low noise signal conditioning and data acquisition.

Depending on the specific measurements mission profile data recording can be performed either at request, on a time scheduled way or on event. The duration of each recorded snippet can also be defined.

The acoustic assessment is performed with a dual frequency $(90 / 300 \mathrm{KHz})$ echo sounder with a $50 \mathrm{~m}$ of range. The simple echosounder solution was chosen due to overall cost and size restrictions (when comparing with more resolution or imaging solutions).

\section{DATA ANALYSIS AND MODELING PLATFORM}

MarinEye includes a centralized data base infra-structure that will aggregate the disparate data sources (physical, chemical, biological) that will be collected by the multitrophic prototype. This data base feeds a platform for data visualization and summarization that can provide synthetic summaries of the main events of the system in a way that facilitates the analysis of researchers. Moreover, the platform also implements several modeling tools that have as main goal to uncover unsuspected and useful patterns that may exist on the physico-chemical and biological data sets generated by the multitrophic system with the goal of providing some understanding about how organisms interact with their environment and with each other, and how these interactions influence ecosystem stability

\section{CONCLUSIONS}

Marine organisms exert tremendous power over the planet and linking the organisms to their environments by monitoring biological variables integrated in contextualized environmental data (physical-chemical variables) is essential to evaluate the environmental status of marine ecosystems.

MarinEye will combine sensors (targeting biogeochemistry), fraction filtration systems (targeting prokaryotes and unicelular eukaryotes), imaging (targeting ictio-, zoo- and phytoplankton), sonar (targeting fish) and acoustic (targeting mammals) technologies in a modular, compact system that can be deployed 
on fixed and mobile platforms, providing key data to respond to time sensitive environmental issues.

The implementation of MarinEye will generate data that will give an integrated view of the different compartments of the main pelagic oceanic ecosystem, combining physical, chemical, and different biological levels. Such information will be synchronized in time and space what will allow a continuous monitoring of major ecosystem processes and thus helping deriving accurate multimetric indicators to effectively assess the environmental status of marine ecosystems. MarinEye will allow tackling the enormous knowledge gap in understanding the dynamics of the different biological pelagic compartments, constituting a valuable contribution to develop marine strategies, namely the environmental monitoring of marine coastal, remote and ocean protected areas.

\section{REFERENCES}

[1] F. Muller-Karger, et al. , "A Framework for a Marine Biodiversity Observing Network Within Changing Continental Shelf Seascapes," Oceanography, vol. 27, no. 2, pp. 18-23, 2014.

[2] H. Claustre, et. al., "Guidelines towards an integrated ocean observation system for ecosystems and biogeochemical cycles." 2009.

[3] C. R. Barnes, "Building the World's First Regional Cabled Ocean Observatory (NEPTUNE): Realities, Challenges and Opportunities," in Oceans 2007, 2007, pp. 1-8.

[4] J. Moloney, C. Hillis, X. Mouy, I. Urazghildiiev, and T. Dakin, "Autonomous Multichannel Acoustic Recorders on the VENUS Ocean Observatory," in 2014 Oceans St. John's, 2014, pp. 1-6.

[5] T. B. Curtin, J. G. Bellingham, J. Catipovic, and D. Webb, "Autonomous oceanographic sampling networks," Oceanography, vol. 6, pp. 86-94, 1993.

[6] N. A. Cruz and A. C. Matos, "The MARES AUV, a Modular Autonomous Robot for Environment Sampling," in OCEANS 2008, 2008, pp. 1-6.

[7] H. Ferreira, C. Almeida, A. Martins, J. Almeida, N. Dias, A. Dias, and E. Silva, "Autonomous bathymetry for risk assessment with ROAZ robotic surface vehicle," in OCEANS 2009-EUROPE, 2009, pp. 1-6.
[8] A. Bode, J. Bueno, Á. López-urrutia, F. Villate, I. Uriarte, A. Iriarte, M. T. Alvarez-ossorio, A. Miranda, G. Casas, A. M. P. Santos, M. A. Chícharo, J. Cruz, and L. Chícharo, "Zooplankton of the bay of Biscay and Iberian shelf," 2011.

[9] P. Otero, M. Ruiz-Villarreal, L. Garcia-Garcia, M. Marta-Almeida, M. Cobas, G. Gonzalez-Nuevo, and J. M. Cabanas, "Walking on the sea side: Modeling and observational efforts of the Iberian Margin Ocean Observatory (RAIA)," in OCEANS 2011 IEEE - Spain, 2011, pp. 1-6.

[10] N. Gruber, A. Körtzinger, et. al., "Warming up , turning sour, losing breath: an integrated observing system for ocean biogeochemistry at a time of change," in Proceedings of OceanObs'09: Sustained Ocean Observations and Information for Society, 2009, vol. 369, no. 1943.

[11] A. C. Trembanis, C. Cary, V. Schmidt, D. Clarke, T. Crees, and E. Jackson, "Modular autonomous biosampler (MAB) - A prototype system for distinct biological size-class sampling and preservation," in 2012 Oceans, 2012, pp. 1-6.

[12] C. D. Taylor, K. W. Doherty, S. J. Molyneaux, A. T. Morrison, J. D. Billings, I. B. Engstrom, D. W. Pfitsch, and S. Honjo, "Autonomous Microbial Sampler (AMS), a device for the uncontaminated collection of multiple microbial samples from submarine hydrothermal vents and other aquatic environments," Deep Sea Res. Part I Oceanogr. Res. Pap., vol. 53, no. 5, pp. 894-916, May 2006.

[13] C. A. Scholin, E. I. Massion, D. K. Wright, D. E. Cline, E. Mellinger, and M. Brown, "Aquatic autosampler device," 6187530, 2001.

[14] A. Kopf, et. al. , "The ocean sampling day consortium.," Gigascience, vol. 4, p. 27, Jan. 2015.

[15] M. Benfield, P. Grosjean, P. Culverhouse, X. Irigolen, M. Sieracki, A. Lopez-Urrutia, H. Dam, Q. Hu, C. Davis, A. Hanson, C. Pilskaln, E. Riseman, H. Schulz, P. Utgoff, and G. Gorsky, "RAPID: Research on Automated Plankton Identification," Oceanography, vol. 20, no. 2, pp. 172-187, 2007.

[16] R. K. Cowen and C. M. Guigand, "In situ Ichthyoplankton Imaging System (ISIIS): system design and preliminary results," Limnol. Oceanogr. Methods, vol. 6, pp. 126-132, 2008. 


\section{ACKNOWLEDGEMENTS}

The authors acknowledge the financial support from Iceland, Liechtenstein and Norway through the EEA Grants (PT02_Aviso4_0017). C. Magalhães and M.F. Carvalho acknowledge Investigator FCT program supported by Fundação para a Ciência e a Tecnologia (FCT), Fundo Social Europeu (FSE) and Programa Operacional Potencial Humano. This work was also partially funded by the ERDF - European Regional Development Fund through the Operational Programme for Competitiveness and Internationalisation - COMPETE 2020 Programme within project «POCI-01-0145-FEDER-006961», and by National Funds through the FCT - Fundação para a Ciência e a Tecnologia (Portuguese Foundation for Science and Technology) as part of project UID/EEA/50014/2013. 